\title{
Impaired nuclear export of tumor-derived c-terminal truncated cyclin D1 mutant in ESCC cancer
}

\author{
MEILI HAO $^{1 *}$, XIANGMEI CHEN ${ }^{2 *}$, TING ZHANG $^{2}$, TAO SHEN $^{2}$, \\ QING XIE ${ }^{2}$, XIUJUAN XING ${ }^{2}$, HONGXI GU ${ }^{1}$ and FENGMIN LU ${ }^{2}$ \\ ${ }^{1}$ Department of Microbiology, Harbin Medical University, Harbin, Heilongjiang 150081; ${ }^{2}$ Department of Microbiology \\ and Infectious Disease Center, Peking University Health Science Center, Beijing 100191, P.R. China
}

Received April 6, 2011; Accepted August 17, 2011

DOI: $10.3892 / \mathrm{ol} .2011 .404$

\begin{abstract}
Cyclin D1 is a significant regulator of the G1- to S-phase transition and is often aberrant in human tumors of various origins. Although cancer-derived cyclin D1 mutants are potent oncogenes in vitro and in vivo, the mechanisms by which they contribute to neoplasia remaind to be elucidated. We previously identified a cyclin D1 mutation $(\Delta 266-295)$ in esophageal cancer with deleted codons from 266 to 295 of wild-type cyclin D1, the critical $\mathrm{COOH}$-terminal regulatory sequences necessary for cyclin D1 nuclear export. In the present study, this cancer-derived cyclin D1- $\Delta 266-295$ was shown to be a constitutively nuclear cyclin D1 protein with a significantly increased oncogenic potential. Moreover, the cancer-derived cyclin D1- $\Delta 266-295$ mutant was found to retain its ability to bind to and activate CDK4, which in turn phosphorylates and inactivates the $\mathrm{pRb}$ protein and promotes cell cycle progression. In comparison to wild-type cyclin D1a, D1- $\Delta 266-295$ exhibited enforced nuclear accumulation. In addition, the transient transfection and ectopic expression of this nuclear localized D1- $\Delta 266-295$ up-regulated endogenous Notch1 expression, indicating that the mutant retained its ability as a transcriptional regulator. Furthermore, data from the flow cytometry assay showed that D1- $\Delta 266-295$ fractionally increased $>4 \mathrm{~N}$ cell accumulation, and further analysis suggested the retriggering of DNA replication relevant to its inhibition of Cdt1 proteolysis. Therefore, the inappropriate nuclear localization of this cyclin D1 mutant
\end{abstract}

Correspondence to: Dr Xiangmei Chen, Department of Microbiology and Infectious Disease Center, Peking University Health Science Center, Beijing 100191, P.R. China

E-mail: xm_chen6176@bjmu.edu.cn

Dr Hongxi Gu, Department of Microbiology, Harbin Medical University, Harbin, Heilongjiang 150081, P.R. China

E-mail: hxgu2432@163.com

*Contributed equally

Key words: cyclin D1, cyclin-dependent kinase 4, nuclear export, cell proliferation, cancer may interfere with DNA replication in cultured cells, thereby contributing to genomic instability.

\section{Introduction}

Cyclin D1 is a significant regulator of cell cycle progression in numerous cell types. Cyclin D1 elicits its pro-proliferative function early in the G1 phase, as it is capable of activating cyclin-dependent kinases (CDKs) 4 or 6 . Active CDK4/6cyclin D1 complexes phosphorylate and inactivate the retinoblastoma protein $(\mathrm{Rb})$, which is critical for modulating G1- to $\mathrm{S}$-phase progression, and in this manner promote cell proliferation (1-3). In addition to its well-established cell cycle roles, cyclin D1 is involved in CDK-independent function in transcription by acting as a molecular bridge between DNA-bound transcription factors and chromatin-modifying enzymes (4-6).

Cyclin D1 expression is regulated mainly by extracellular mitogenic and oncogenic signals, allowing cyclin D1 to serve as a mediator of growth factor signaling and cell cycle progression (7). It is therefore unsurprising that cyclin D1 is often deregulated in tumors of various origins (8). The overexpression of cyclin D1 caused by gene amplification is observed in several carcinomas, including those of the esophagus, head and neck, breast and colon (9-16). Notably, unlike strong oncogenes, such as Ras, the overexpression of cyclin D1 alone is not capable of transforming immortalized murine fibroblasts in vitro (17). Furthermore, whereas the overexpression of cyclin D1 is considered to be the initial genetic trigger in mantle cell lymphoma, targeted expression of wild-type cyclin D1 in lymphoid cells does not result in a tumor-prone phenotype in transgenic mice $(18,19)$, thereby challenging the notion that cyclin D1 is an oncogene.

Findings of a previous study showed that the inhibition of cyclin D1 nuclear export during the S phase unmasks its neoplastic potential (20). Phosphorylation of cyclin D1 at threonine 286 (Thr286) is required for its nuclear export and degradation in the cytoplasm $(17,21,22)$. This phosphorylation is mediated by glycogen synthase kinase $3 \beta$ (GSK-3 $\beta$ ) and is greatly enhanced by the binding of cyclin D1 to CDK4 (23). The expression of the artificially engineered cyclins D1-T286A or D1b, a naturally occurring alternative splice variant of cyclin D1, cannot be phosphorylated by GSK-3 $\beta$ and are stabilized in the nucleus. Moreover, these variants are 
capable of transforming murine fibroblasts in the absence of a collaborating oncogene $(21,24)$. Furthermore, constitutively nuclear cyclin D1 mutants have been identified in certain solid tissue tumors, such as esophageal and endometrial cancers, that promote tumorigenesis in transgenic mice $(25,26)$. These results suggested that the deregulation of cyclin D1 nuclear export is a tumor-initiating event. Although cancer-derived cyclin D1 mutants are potent oncogenes in vitro and in vivo, the mechanisms by which they contribute to neoplasia remain to be elucidated. Among the mutations detected in esophageal cancers was a deletion encompassing codons 266-295 of cyclin D1 ( $\Delta 266-295)$. As with cyclin D1b, this cancer-derived D1- $\Delta 266-295$ mutant possesses the cyclin box required for CDK binding and enzymatic activity, but lacks the PEST destruction box and Thr286, which are crucial to the promotion of the nuclear export of cyclin D1 and its turnover (14). Using function analysis, the purpose of this study was to show that this cancer-derived deletion mutant D1- $\Delta 266-295$ retained its ability to support CDK4 catalytic activity, and was characterized by constitutive nuclear localization, thereby contributing to increasing cyclin D1 oncogenic capacity. Consequently, insight would be gained as to the mechanism involved in such mutations contributing to the genesis and progression of neoplastic growth.

\section{Materials and methods}

Cell culture conditions and transfections. NIH3T3 and HepG2 cells were cultured in DMEM supplemented with $2 \mathrm{mM}$ L-glutamine, 10\% FBS and antibiotics (Gibco, Carlsbad, CA, USA). KYSE510 cells were grown with RPMI-1640 supplemented with $2 \mathrm{mM}$ L-glutamine, 10\% FBS and antibiotics. Transient transfections were performed following the manufacturer's instructions using Lipofectamine Plus (Invitrogen, Carlsbad, CA, USA). Cyclin D1- 2 266-295 plasmid was engineered using $\mathrm{pFlex}$-cyclin Dla vector as a template with primers 5'-GGTGGTGATTACAAAGATGACGACGATAAG-3' (forward) and 5'-ACGGAATTCAGTTCTGCTGGGCCTG-3' (reverse). The PCR products were purified with Wizard $^{\circledR} \mathrm{SV}$ Gel and the PCR clean-up system (Promega, Madison, WI, USA) and inserted into the pFlex vector as EcoRI fragments as previously described for cyclin Dla (24) to generate Flagtagged molecules.

Immunoblotting and immunoprecipitation assays. For direct Western blot analysis, cells were washed with pre-chilled phosphate-buffered saline (PBS) and lysed in lysis buffer containing $0.1 \mathrm{~mol} / 1 \mathrm{NaCl}, 0.01 \mathrm{~mol} / 1$ Tris-Cl (pH 7.6), $0.1 \%$ SDS, 20 mmol/1 EDTA (pH 8.0), 10\% Triton X-100, 1 mmol/l PMSF, $10 \mu \mathrm{g} / \mathrm{ml}$ aprotinin and $10 \mu \mathrm{g} / \mathrm{ml}$ leupeptin. Total cell protein was resolved on denaturing polyacrylamide gels, transferred to nitrocellulose membranes (Bio-Rad, Hercules, CA, USA), and blotted with antibodies obtained for cyclin D1a (SC-8396; Santa Cruz Biotechnology, Santa Cruz, CA, USA), M2 (F1804; Sigma, St. Louis, MO, USA), Rb (9309L; CST), phospho-Rb-Ser780 (9307S; CST), Myc (BM2901-02; Biomiga), Tublin (sc-9104; Santa Cruz Biotechnology) and CDK4 (sc-26; Santa Cruz Biotechnology). Protein-antibody complexes were visualized either by using secondary antibodies (goat-anti-rabbit IgG or goat-anti-mouse IgG) followed by enhanced chemiluminescence, or by using secondary antibodies conjugated with Cy5.5 (Amersham Pharmacia Biotech, Piscataway, NJ, USA) and visualized using the LI-COR Odyssey IR Imaging System (LI-COR Biosciences, Lincoln, NE, USA).

For co-immunoprecipitation (Co-IP), cells were lysed in IP buffer containing $20 \mathrm{mM}$ Tris- $\mathrm{HCl}(\mathrm{pH} 7.4), 150 \mathrm{mM}$ $\mathrm{NaCl}, 1 \mathrm{mM}$ EDTA, $1 \%$ Triton X-100, 10\% glycerol, $1 \mathrm{mmol} / \mathrm{l}$ PMSF, $10 \mu \mathrm{g} / \mathrm{ml}$ aprotinin and $10 \mu \mathrm{g} / \mathrm{ml}$ leupeptin, and were centrifuged for $30 \mathrm{~min}$ at $10,000 \mathrm{x} \mathrm{g}$ at $4^{\circ} \mathrm{C}$. The supernatant was incubated with primary antibody overnight at $4^{\circ} \mathrm{C}$. The immunocomplexes were bound to protein- $\mathrm{G}$ sepharose $4 \mathrm{~B}$ (Pharmacia, USA) for $1 \mathrm{~h}$ at $4^{\circ} \mathrm{C}$ and washed three times with IP buffer. Proteins bound to the protein-G sepharose 4B were eluted by adding Laemli-SDS sample buffer and then boiling for $5 \mathrm{~min}$. Following centrifugation at $10,000 \mathrm{x} \mathrm{g}$ for $2 \mathrm{~min}$, the supernatant was analyzed by immunoblotting.

Protein turnover analysis. To measure the turnover rate of cyclin D1 protein, Flag-tagged wild-type or mutant cyclin D1 plasmids were transiently transfected into NIH-3T3 cell lines. Forty-eight hours after transfection, cycloheximide $(50 \mu \mathrm{g} / \mathrm{ml}$; Sigma) was added to block new protein synthesis. Cells were then harvested at 0,30,60, 90, 120 and 180-min intervals following treatment with cycloheximide. Cell lysates were resolved by SDS-PAGE and the rate of cyclin D1 decay was then assayed by direct Western blotting.

Indirect immunofluorescence assays. NIH-3T3 cells overexpressing either Flag-tagged wild-type or mutant cyclin D1 were seeded on glass coverslips. Cells were then fixed using either $3 \%$ paraformaldehyde or methanol-acetone (1:1) as previously described (24). Visualization of cyclin D1 was achieved with the Flag-specific M2 monoclonal antibody. Secondary TRITC-conjugated anti-mouse antibody (IgG; Sigma) staining was performed for $60 \mathrm{~min}$ at room temperature under moisture. DNA was visualized using Hoechst 33258 dye at a 1:1,000 dilution. Coverslips were mounted on glass slides with vectashield medium (Vector Laboratories Inc., Burlingame, CA, USA) and visualized under a fluorescence microscope.

Real-time quantitative PCR analysis of gene expression. RNA isolation was performed using standard protocols. cDNA was prepared by reverse transcription (SuperScript, Invitrogen). Real-time PCR was performed on a Roche LightCycler ${ }^{\circledR} 480$ sequence detection system using LightCycler 480 SYBR-Green I Master mix (Roche, Indianapolis, IN, USA). Amplification of the housekeeping gene GAPDH was performed to standardize the amount of sample RNA. Primers used for the detection of human Notch1 were 5'-CACTGATCCTGGCTGCC CGC-3' (forward); and 5'-CAGCAGCACCTTGGCGGTCT-3' (reverse). Primers used for the detection of human GAPDH were 5'-GAGTCAACGGATTTGGTAGT-3' (forward); and 5'-TTG ATTTTGGAGGGATCTCG-3' (reverse). The thermal cycler conditions were as follows: $2 \mathrm{~min}$ at $50^{\circ} \mathrm{C}$, hold for $10 \mathrm{~min}$ at $95^{\circ} \mathrm{C}$, followed by two-step PCR for 40 cycles of $95^{\circ} \mathrm{C}$ for $15 \mathrm{sec}$ followed by $60^{\circ} \mathrm{C}$ for $1 \mathrm{~min}$. After normalization to the GAPDH, the relative quantification of gene expression was performed using the $2^{-\Delta \mathrm{Ct}}$ method, and each experiment was carried out in triplicate. 
A

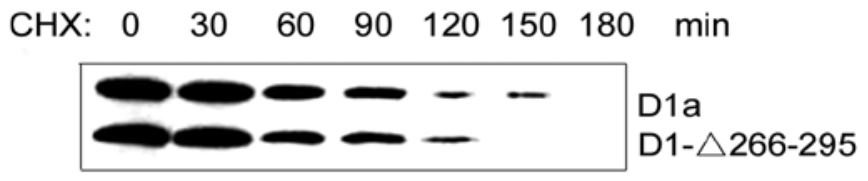

C

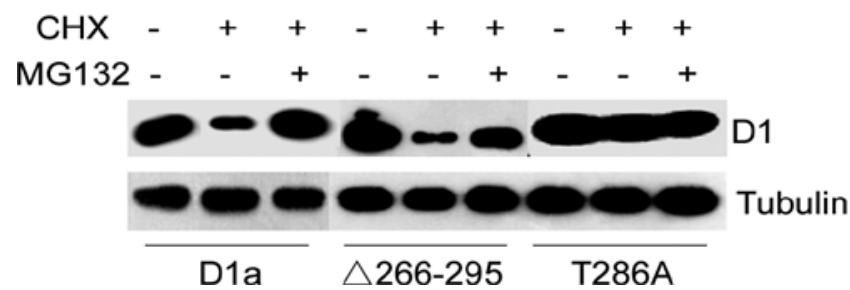

B

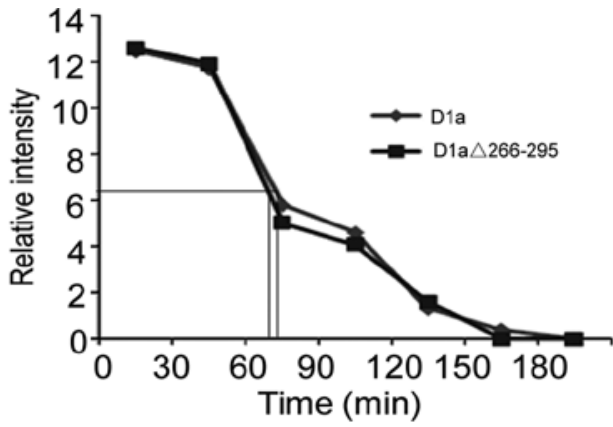

D

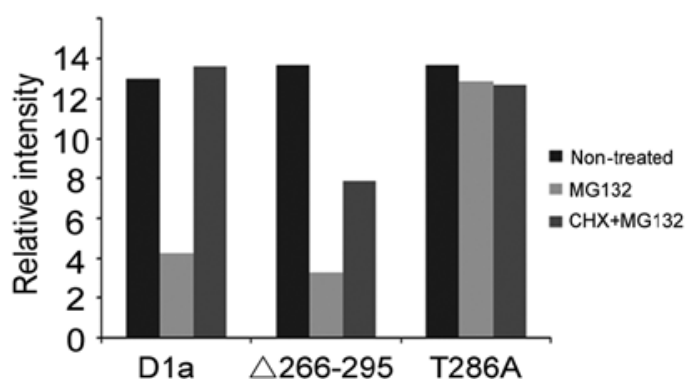

Figure 1. Cyclin D1- $\Delta 266-295$ protein half-life unchanged. (A) NIH-3T3 cells transfected with vectors encoding Flag-tagged cyclin D1a (pFlex-D1a) and D1- $\Delta$ 266-295 (Flex-D1- $\Delta 266-295)$ were treated with cycloheximide $(50 \mu \mathrm{g} / \mathrm{ml})$ for the indicated intervals. Lysates prepared from the respective cell lines were processed for Western blot analysis using the M2 antibody. (B) The half-lives of cyclins D1a and D1- $\Delta 266-295$ were deduced from the curves analyzed with Quantity One software (Bio-Rad). (C) Lysates of NIH-3T3 cell lines overexpressing the indicated Flag-tagged proteins, treated with cycloheximide or MG132 for $6 \mathrm{~h}$, were blotted with the M2 antibody. (D) Quantification of Flag-tagged D1 expression shown in (C).

Flow cytometry. For cell cycle analysis, cells were harvested by trypsinization and fixed with $70 \%$ ethanol at $4^{\circ} \mathrm{C}$ overnight. The fixed cells were rinsed twice with PBS and resuspended in propidium iodine (PI) solution, including $50 \mu \mathrm{g} / \mathrm{ml} \mathrm{PI}$ and $50 \mu \mathrm{g} / \mathrm{ml}$ RNaseA (Sigma) in PBS without calcium and magnesium, and incubated at $37^{\circ} \mathrm{C}$ for $30 \mathrm{~min}$ in the dark. The fluorescence of the cells was measured by a FACSCalibur system (Nippon Becton Dickinson, Tokyo, Japan), and the percentages of cells in the G1, S and G2/M phases were determined by the ModFit program (Nippon Becton Dickinson). Flow cytometry analysis was repeated three times with the variation in results $<20 \%$. For analysis of $>4 \mathrm{~N}$ DNA content, cells were harvested, fixed with ethanol and stained with PI staining buffer. The number of cells with $>4 \mathrm{~N}$ DNA content were counted, due to the firing of replication origins more than once per cell cycle.

\section{Results}

Turnover of cyclin D1-4266-295. Phosphorylation of Thr286 not only directs cyclin D1 nuclear export, but also promotes the rapid proteasome-dependent destruction of cyclin D1. We therefore predicted that D1- $\Delta 266-295$ would be refractory to proteasomal degradation and exhibit an extended half-life relative to wild-type cyclin D1. To functionally characterize the tumor-derived cyclin D1 deletion D1- $\Delta 266-295$ protein, wild-type D1a and D1- $\Delta 266-295$ were engineered to encode a NH2-terminal Flag-epitope tag (pFlex-D1- $\Delta 266-295$ or pFlex-D1a). The turnover of D1- $\Delta 266-295$ vs. D1a was initially examined in NIH-3T3 cells engineered to express Flag-D1a and Flag-D1- $\Delta 266-295$ ectopically. However, inconsistent with the loss of Thr286 phosphorylation and increased nuclear retention, cyclin D1- $\Delta 266-295$ exhibited an almost identical half-life to wild-type cyclin D1 (Fig. 1A). The halflife was $\sim 75 \mathrm{~min}$ for cyclin D1a and $70 \mathrm{~min}$ for D1- $\Delta 266-295$ (Fig. 1B). The absence of the degradation box and Thr286 had no major effect on the turnover of cyclin D1.

We then investigated whether the degradation of cyclin D1- $\Delta 266-295$ resulted from proteolysis via the $26 \mathrm{~S}$ proteasome. To this end, specific inhibitors cycloheximide (CHX) and MG132 were exploited to block a new protein synthesis and to inhibit proteasome-dependent proteolysis, respectively. As shown in Fig. 1C, the treatment of transfected cells with $\mathrm{CHX}$ for $6 \mathrm{~h}$ resulted in a reduced level of cyclins D1a and D1- $\Delta 266-295$ (lane 2 vs. lane 1, lane 5 vs. lane 4, Fig. 1C). Once treated with CHX along with MG132, the protein levels of cyclins D1a and D1- $\Delta 266-295$ in transfected cells were significantly higher than those in only $\mathrm{CHX}$-treated cells (lane 3 vs. lane 2, lane 6 vs. lane 5, Fig. 1C). As for cyclin D1-T286A-transfected cells, treatment with CHX only, or with both $\mathrm{CHX}$ and MG132, did not change the protein level of cyclin D1-T286A (lane 7 vs. lanes 8 and 9, Fig. 1C). Taken together, these data indicate that cyclin D1- $\Delta 266-295$ is degraded by the $26 \mathrm{~S}$ proteasome in the same manner as cyclin D1a, while cyclin D1-T286A is refractory. Furthermore, the proteasome inhibitor MG132 significantly increased the cyclin D1a protein level compared to that of cyclin D1- $\Delta 266$ 295 (Fig. 1D), suggesting that there is another degradation mechanism for cyclin D1- $\Delta 266-295$.

Subcellular localization of cyclin D1-4266-295. Since cyclin D1- $\Delta 266-295$ lacks the GSK-3 $\beta$ phosphorylation site, we reasoned that it may be refractory to the nuclear export directed by GSK-3 $\beta$ and CRM1, and exhibit a nuclear localization pattern. To test this hypothesis, D1- $\Delta 266-295$ and D1a localization was examined by indirect cyto-immunofluorescence 
A
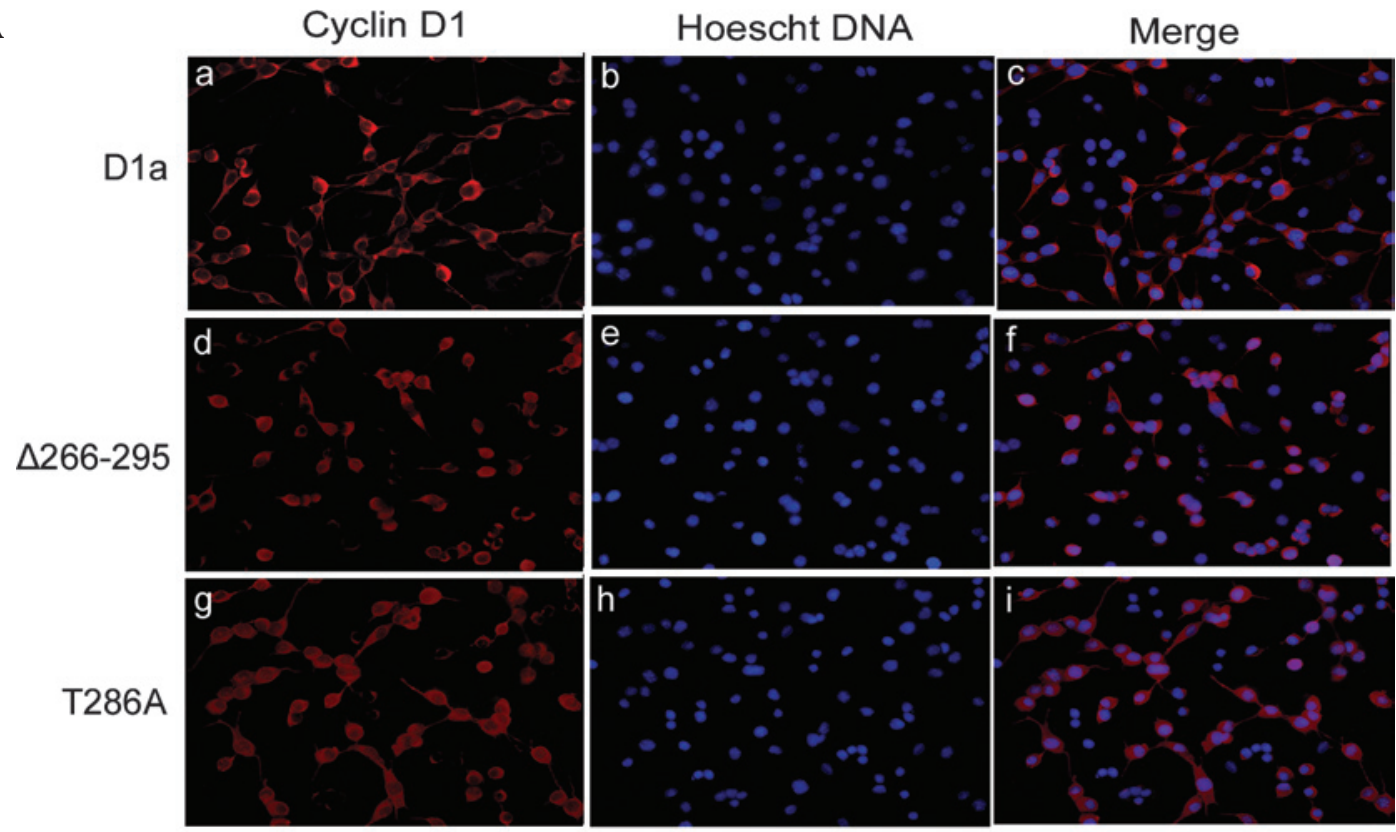

$\mathbf{B}$

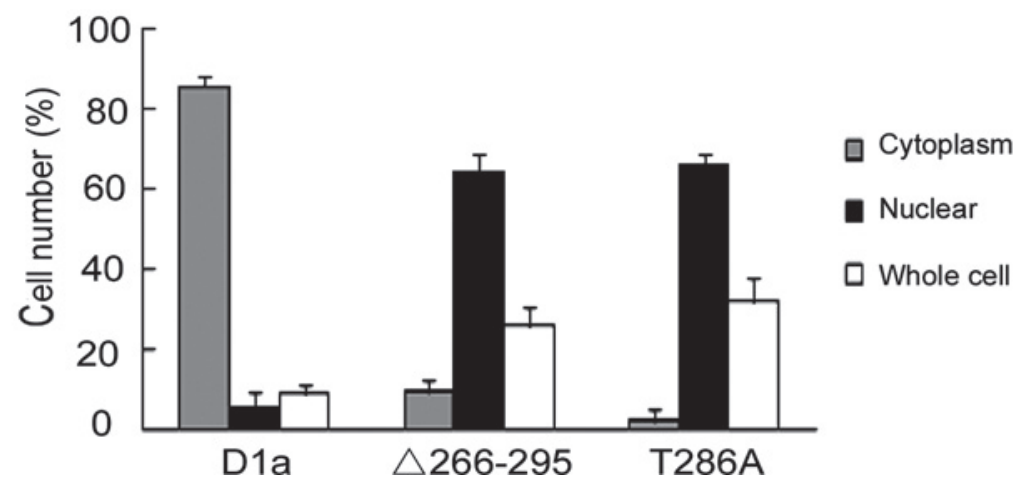

Figure 2. Cyclin D1- $\Delta 266-295$ behaves in the same manner as the D1-T286A mutant and remains nuclear. (A) NIH-3T3 cells were transfected with (a-c) pFlex-D1a, (d-f) pFlex-D1- $\Delta$ 266-295 or (g-i) pFlex-D1-T286A. The subcellular localization of cyclin D1 protein in NIH-3T3 cell lines was determined by immunofluorescence using M2 antibody followed by TRITC-conjugated anti-mouse secondary antibody (red). Corresponding Hoechst DNA staining is shown (blue) and a merged view (merge) of both channels (red/blue). (B) Quantification of immunofluorescence shown in (A). Bars are the standard deviation between independent transfections.

staining in asynchronous NIH3T3 cells. Staining with the M2 antibody showed an apparent cytoplasmic localization of cyclin Dla with little nuclear overlap as expected (Fig. 2A, a-c). By contrast, cyclin D1- $\Delta 266-295$ exhibited exclusively nuclear localization patterns (Fig. 2A, d-f), behaving in the same manner as the previously reported D1-T286A mutant, remaining nuclear throughout the interphase (Fig. 2A, g-i). Quantification showed that D1a was distributed predominantly in the cytoplasm and that only 5\% of cells were nuclearpositive, whereas the distribution of D1- $\Delta 266-295$ and D1-T286A was predominantly nuclear at 63 and $66 \%$, respectively (Fig. 2B).

Analysis of the cyclin D1-4266-295/CDK4 complex formation and cyclin D1-4266-295 transcriptional function. $\mathrm{pRb}$ is the critical target of the cyclin D1/CDK4 complex, and the cell cycle transition from the G1- to S-phase requires the temporal activation of cyclin D1/CDK4 and subsequent Rb phosphorylation. To investigate the ability of cyclin D1- $\Delta 266-295$ to form binary complexes with CDK4, co-immunoprecipitation experiments were performed in NIH-3T3 cells, and cyclin D1/
CDK4 complexes were isolated from whole cell lysates using CDK4-specific antibody. The presence of D1a, D1- $\Delta 266-295$ and D1-T286A was determined by immunoblotting with the M2 antibody. As shown in Fig. 3A, D1- $2266-295$ retained the ability to bind to CDK4 to the same extent as D1a and D1-T286A, as CDK4 was expressed at the same levels in each precipitate (Fig. 3A, middle). A similar expression of the various types of cyclin D1 in NIH-3T3 cells was confirmed with total protein extracts (input, lanes 2 and 3 vs. lane 4). To evaluate the ability of CDK4/D1- $\Delta 266-295$ complexes to mediate Rb phosphorylation, lysates prepared from NIH-3T3 cells transfected with cyclin D1 mutations were subjected to Western blot analysis with the antibody specific for phosphorserine at amino acid 780 (pRb780Ser), which is a site of CDK4-mediated phosphorylation on Rb. Flag-D1a and FlagD1- $\Delta 266-295$ efficiently promoted Rb780Ser phosphorylation when overexpressed in NIH-3T3 cells (Fig. 3B, top; lane 1 vs. lanes 2 and 3).

Cyclin D1 also acts as a transcriptional modulator by regulating the activity of several transcription factors. Cyclin D1 has been shown to bind the upstream regulatory region of the 
A

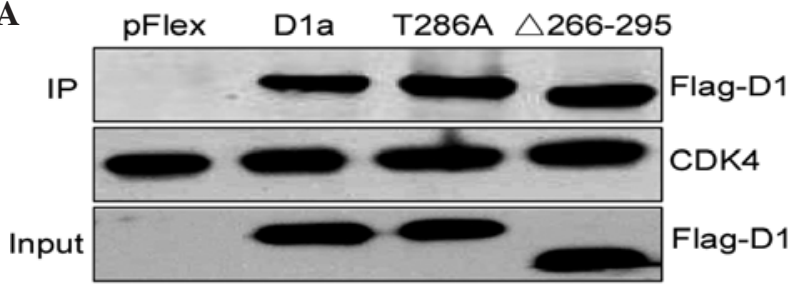

B

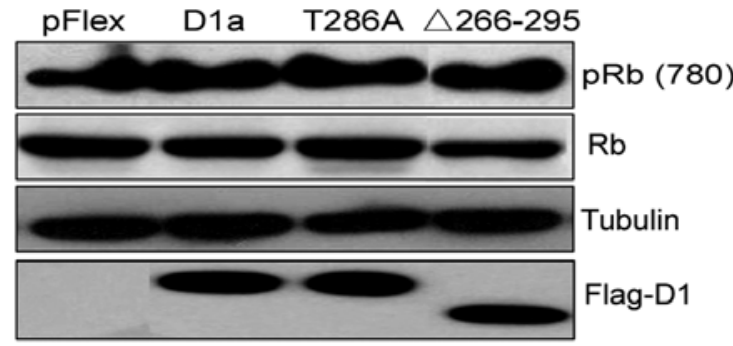

C

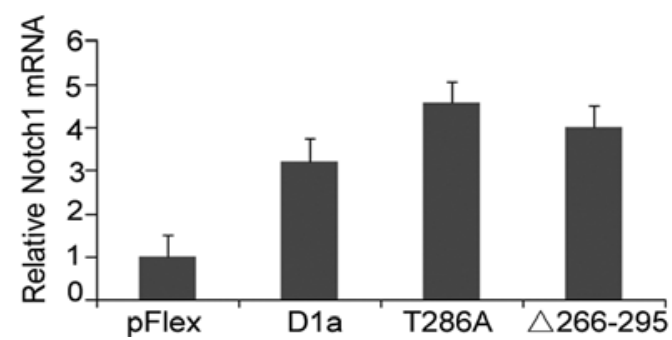

Figure 3. Cyclin D1- $\Delta 266-295$ binds to CDK4, and D1- 2 266-295/CDK4 complexes were catalytically active. (A) Cell lysates were prepared from NIH-3T3 cells transfected with vectors pFlex (negative control), pFlex-D1a, pFlex-D1-T286A or pFlex-D1- $\Delta 266-295$, and were precipitated with antibodies directed towards the CDK4. Precipitated proteins were assessed by Western blot analysis with antibodies directed towards the Flag epitope (M2) (top panel) or anti-CDK4 (second panel). One tenth of the total cell lysates was subjected to direct Western blot analysis to confirm the expression levels of each cyclin D1 isoform (third panel). (B) Whole cell extracts were obtained from NIH-3T3 cells transfected with the indicated cyclin D1, separated on SDS-PAGE and sequentially immunoblotted with anti-Rb and anti-pRb (780). For loading controls, blots were also revealed with $\mathrm{M} 2 \mathrm{Ab}$ and anti- $\beta$-tubulin $\mathrm{Ab}$ antibodies. (C) Levels of Notch1 mRNA expression in KYSE510 cells, quantified by real-time PCR following the overexpression of cyclin D1 viarants. The fold-difference compared to cells transfected with the control vector is shown. Error bars are standard deviation.

Notch1 gene where it serves to recruit CBP histone acetyltransferase, and increases Notch1 mRNA levels in vivo. We then determined whether D1- $\Delta 266-295$ mutation disrupts this transcriptional ability by assessing the mRNA expression of the Notch1 gene in the KYSE510 human esophageal carcinoma cells overexpressing the respective cyclin D1 mutant. Real-time PCR analysis results showed that D1- 2 266-295 increased Notch 1 mRNA levels in a similar manner to the wild-type cyclin D1 and T286A mutations, demonstrating that the transcriptional ability of cyclin D1- $\Delta 266-295$ was not eradicated (Fig. 3C). These results emphasized the retained structural and functional integrity of D1- $\Delta 266-295$.

Effects of cyclin D1-4266-295 on cell proliferation. The effects of D1- $\Delta 266-295$ on cell proliferation were investigated and compared to those of cyclins D1a and D1-T286A. To investigate the effects of cyclin D1 on cell cycle progression, the human esophageal carcinoma KYSE510 cells transfected with pFlex (control), pFlex-D1a, pFlex-D1-T286A or pFlex-D1- $\Delta 266-295$, were treated with serum starvation for $24 \mathrm{~h}$. Cells were then harvested and analyzed by flow cytometry. As shown in Fig. 4A and B, with serum, the fractions of cells in the $\mathrm{G} 0 / \mathrm{G} 1, \mathrm{~S}$ or $\mathrm{G} 2 / \mathrm{M}$ phases were 68.67 , 25.65 and $5.68 \%$, respectively, for Flex-KYSE510 (control). Following $24 \mathrm{~h}$ of serum deprivation, the corresponding fractions yielded were $80.51,13.9$ and $5.59 \%$, respectively, indicating that serum starvation blocked cells in the G1 phase as demonstrated by the increase in the G0/G1 cell fraction (80.51 vs. $68.67 \%)$. In comparison to Flex-KYSE510 cells, even following $24 \mathrm{~h}$ of serum starvation, only $58.02 \%$ of D1- $\Delta 266-295-K Y S E 510$ cells were observed to be in the G0/ G1 phase, whereas as many as $34.04 \%$ were in the $S$ phase, a similar level to that observed with Flex-KYSE510 control with serum. This finding suggested that D1- $\Delta 266-295$ stimulates the KYSE510 cell cycle progression and accelerates the entry and proportion of cells in the $S$ phase, even without serum stimulation. As expected, D1a-KYSE510 and D1-T286A-KYSE510 cells exhibited similar results to those of D1- $\Delta 266-295-K Y S E 510$, demonstrating that there is no difference in the promotion of cell cycle progression between cyclins D1- $\Delta 266-295$ and D1a, although D1- $\Delta 266-295$ is defective for the $\mathrm{C}$-terminal sequences. To expand our analysis, we also examined the effect of the exogenous expression of D1- $\Delta 266-295$ on the cell cycle distribution of HepG2 cells, a type of hepatic tumor cell line, and similar results as those with KYSE510 were observed (data not shown).

Effects of cyclin D1-4266-295 on DNA re-replication. The increased oncogenicity of constitutively nuclear cyclin D1T286A and transcription isoform cyclin D1b relative to wild-type cyclin D1a suggests that nuclear retention during the $S$ phase is a gain-of-function characteristic. D1- $\Delta 266-295$ also accumulated predominantly in the nucleus of expressing cells. Therefore, we tested whether the D1- $\Delta 266-295 / C D K 4$ kinase would induce the accumulation of cells harboring $>4 \mathrm{~N}$ DNA content as a marker of DNA re-replication. Initially, we assessed the ability of cyclin D1- $\Delta 266-295 / C D K 4$ complexes to drive DNA re-replication in cooperation with Cdt1. HepG2 cells were utilized due to their high efficacy of transfection. Consistent with our hypothesis, the coexpression of Cdt1 along with cyclin D1- $\Delta 266-295$ resulted in a significant increase in the $>4 \mathrm{~N}$ population, similar to D1T286A (Fig. 5A). Cells engineered to overexpress D1- $\Delta 266-295$ exhibited $>18 \%$ of cells with $>4 \mathrm{~N}$ DNA content, and D1T286A exhibited $>26 \%$ of cells with $>4 \mathrm{~N}$, whereas wild-type cyclin Dla in cooperation with Cdt1 only induced a marginal increase in the accumulation of $>4 \mathrm{~N}$ cells compared to the control cells transfected with an empty vector (12.86 vs. $8.67 \%)$. Thus, as in the case of D1T286A, cyclin D1- $\Delta 266-295$ cooperated with Cdt1 to promote cell DNA re-replication.

To further examine the correlation between the nuclear retention of cyclin D1- $\Delta 266-295 / C D K 4$ and Cdt1 accumulation, we determined whether the acute expression of nuclear cyclin D1- $\Delta 266-295$ was capable of suppressing Cdt1 proteolysis. HepG2 cells were transfected with plasmids encoding myc-tagged Cdt1 along with either wild-type or mutant cyclin D1. Cdt1 levels were evaluated in the G1 phase (Cdt1 stable) or the $\mathrm{S}$ phase (Cdt1 unstable) by Western blot analysis. As shown in Fig. 5B and C, Cdt1 levels were 
A
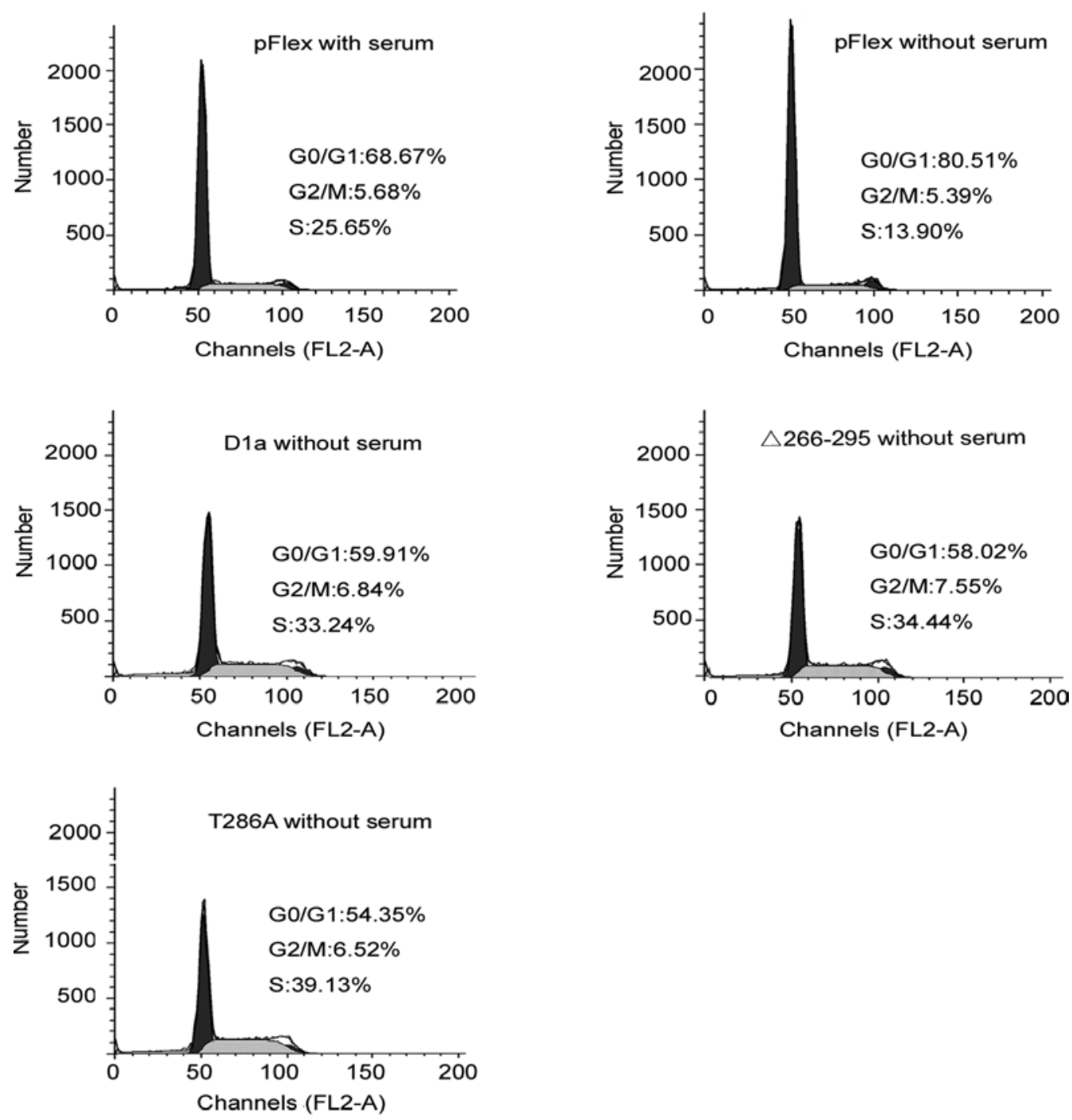

B

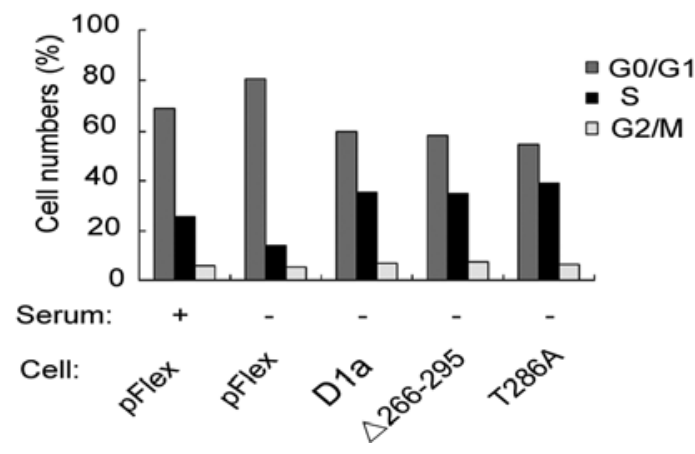

Figure 4. Cyclin D1- $\Delta 266-295$ enhanced the proliferation of KYSE510 cells. (A) KYSE510 cells were transfected with vectors pFlex-D1a, pFlex-D1-T286A or pFlex-D1- $\Delta 266-295$. After $48 \mathrm{~h}$ of transfection, cells were synchronized in G1 by serum starvation for $24 \mathrm{~h}$. Cells were then harvested, stained with propidium iodide and analyzed by flow cytometry. Cells transfected with control vector (pFlex) cultured with or without serum were used as the control. (B) Representative histograms of KYSE510 cells in each cell cycle phase.

markedly reduced in the $\mathrm{S}$ phase in control cells (Fig. 5B, lanes 1 and 2), indicating that the expression of wild-type cyclin D1a did not attenuate Cdt1 degradation (Fig. 5B, lanes 3 and 4). On the other hand, the expression of either D1- $\Delta 266-295$ or D1T286A inhibited S phase-specific Cdt1 loss (Fig. 5B, lanes 5-8), which was consistent with their inability to promote cell DNA re-replication.

\section{Discussion}

Cyclin D1, encoded by the CCND1 gene located on 11q13, plays a significant role in the progression of the cell cycle (27). Cyclin D1 is known to be frequently overexpressed in 40-60\% esophageal cancers, while the frequency of genetic alterations that directly involve the cyclin D1 locus in esophageal 
A
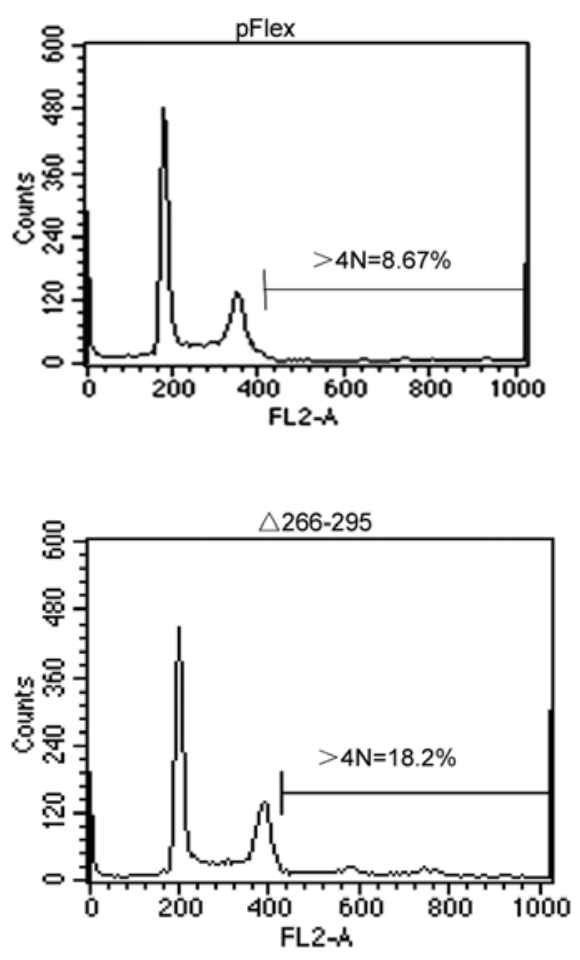

B

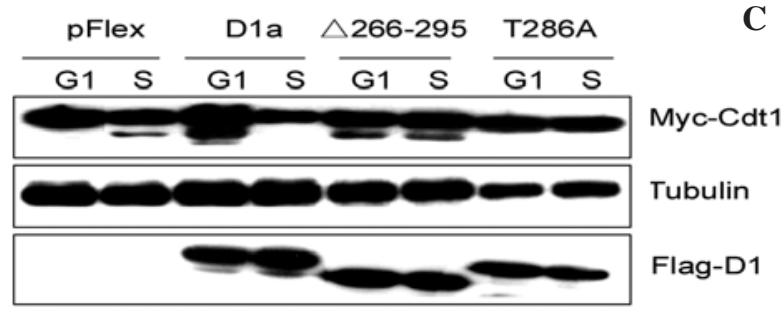

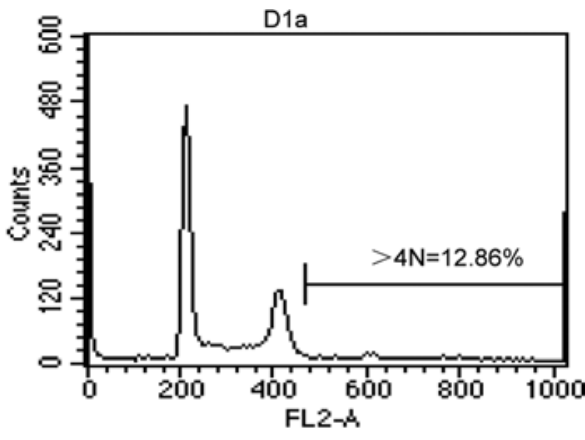
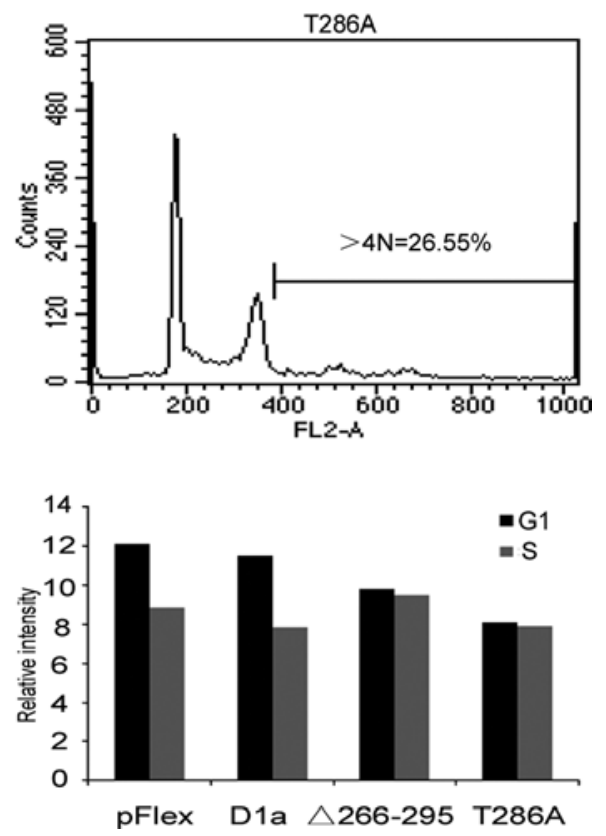

Figure 5. Cdt1, in cooperation with the D1- $\Delta 266$-295-dependent kinase, induces DNA re-replication. (A) HepG2 cells transfected with Cdt1, along with CDK4 and cyclin D1 expression vectors, where indicated, were treated with nocodazole for $14 \mathrm{~h}$, after which cells were shaken off and replated in two plates. The first plate, in complete media, was harvested for $6 \mathrm{~h}$ after release (G1 phase). In the second dish, HU was added and cells were harvested for $15 \mathrm{~h}$ after the shake-off (S phase), stained with propidium iodide and analyzed by flow cytometry. (B) HepG2 cells were transfected with the indicated plasmids and synchronized in the G1 or S phase. Lysates prepared from these cells were analyzed by Western blotting with antibodies directed towards the proteins indicated to the right. (C) The expression levels of Cdt1 analyzed by Quantity One software shown in (B).

cancers is only $10 \%(28,29)$. Thus, predicting frequent alterations in pathways that regulate cyclin D1 protein degradation is significant. Consistent with this postulation, a number of mutations that impair the phosphorylation-dependent nuclear export of cyclin D1 have been identified in esophageal carcinoma and esophageal cancer-derived cell lines (25). Similarly, the cyclin D1 gene in endometrial cancer also possesses mutations or deletions that are expected to affect Thr286 phosphorylation and CRM1 binding (26). These results indicate that mutations promoting constitutive cyclin D1 nuclear localization are likely to be the key oncogenic events.

Cyclin D1 is a short-lived protein. Cyclin D1 is synthesized early in the G1 phase, in response to mitogenic signals, and is then exported from the nucleus and degraded in the cytoplasm during the $\mathrm{S}$ phase, this degradation being required for cell cycle progression (20). The tumor-derived cyclin D1 mutation D1- $\Delta 266-295$ deleted codons from 266 to 295, the critical $\mathrm{COOH}$-terminal regulatory sequences required for cyclin D1 nuclear export. Thus, carboxyl terminal truncated cyclin D1 protein was expected to be a constitutively nuclear cyclin D1 and more stable than cyclin D1a. D1- $\Delta 266-295$ was in fact found to be a constitutively nuclear localized protein. This finding reflects the fact that D1- $\triangle 266-295$ lacks the $\mathrm{COOH}$ terminal sequences targeted by GSK-3 $\beta$ and CRM1. However, we did not find any increase in the half-life of D1- $\Delta 266-295$ indicative of reduced proteolysis. Cyclins D1- $\Delta 266-295$ and Dla have similar rates of protein turnover when expressed in the NIH3T3 cell line. In contrast to another mutant cyclin D1-T286A which demonstrated a 5-fold increase in the measured cyclin D1 half-life (21), nuclear localization of D1- $\Delta 266-295$ does not arrest its degradation. These data suggested that cyclin D1- $\Delta 266-295$ should be more susceptible to nuclear degradation than cyclin D1-T286A.

Similar to our observation on D1- $\Delta 266-295$, cyclin D1b, a naturally occurring alternative splice variant of cyclin D1 lacking the fifth exon, has already been shown to be no more stable than cyclin D1a $(24,30)$. In addition, recent findings have examined the role of GSK-3 $\beta$ in mediating cyclin D1 degradation. Guo et al $(31,32)$ confirmed the role of Thr286 phosphorylation in mediating cyclin D1 degradation in the 
S phase. However, suppressing GSK-3 $\beta$ activity did not have any impact on cyclin D1 phosphorylation or protein levels during the cell cycle. Similarly, GSK-3 $\beta$ localization was not observed to alter with cell cycle progression in MCF-7 breast cancer cells, and inhibition of GSK-3 $\beta$ activity did not completely eradicate cyclin D1 degradation (33). Since cyclin D1 mutants lacking Thr286 remained susceptible to ubiquitination and degradation, our data strongly suggested the existence of a second pathway, which does not require the phosphorylation of Thr286. It is more likely, as previously shown, that the N-terminus, but not the C-terminus, altered cyclin D1 degradation via this pathway $(34,35)$.

Cyclin D1 combines with CDK4 at the cyclin box motif and forms an active complex (36). This complex enters the nucleus and phosphorylates $\mathrm{Rb}$, promoting the release of E2F transcription factors and thus progression from the G1 to $\mathrm{S}$ phase. The cyclin box required for CDK4 interaction is unaffected in D1- $\Delta 266-295$ and, as expected, D1- $\Delta 266-295$ bound to CDK4 and exhibited pRb phosphorylation activity in vivo, in the same manner as the wild-type cyclin D1. In addition, D1- $\Delta 266-295$ retained the transcriptional function on Notch1 gene transcription. Further investigation showed that there was no difference in the promotion of cell cycle progression between cyclins D1- $\Delta 266-295$ and D1a, although D1- $\Delta 266-295$ is defective for the phosphorylation of Thr286 residue in the C-terminal region. Cyclin D1 overexpression was reportedly not sufficient to drive neoplastic growth, while the overexpression of the mutant cyclin D1-T286A induced cell transformation in cell culture and triggered B-cell lymphoma in a mouse model $(17,20)$. Furthermore, transgenic mice that overexpress D1T286A developed mammary adenocarcinoma with a shorter latency relative to mice overexpressing the wild-type cyclin D1 (37). These observations demonstrate that subcellular localization and stabilization of cyclin D1 may exert more profound effects on tumorigenesis than its overexpression. This study provides evidence that cyclin D1- $\Delta 266-295$ may possess oncogenic activity and drive neoplastic growth. This finding suggests that in addition to the well-described G1 functions of cyclin D1 in growth factor signaling and G1- to S-phase progression, the constitutive nuclear retention of mutant cyclin D1 may have additional mechanisms throughout the cell cycle that promote cell transformation.

DNA replication is a highly regulated process that involves numerous licensing and replication factors that cooperate to faithfully replicate DNA during each cell cycle. Loss of proper licensing control results in deregulated DNA replication, including DNA re-replication, which causes genome instability and tumorigenesis (38). Previous studies have shown that inappropriate localization of active cyclin D1/CDK4 complex interferes with the temporal regulation of DNA replication, contributing to genomic instability and neoplastic transformation (39). Nuclear accumulation of the catalytically active mutant cyclin D1T286A/CDK4 complex has been proven to stabilize Cdt1, an origin-licensing factor that is usually degraded during the $\mathrm{S}$ phase to arrest reloading of the replicative MCM helicase. Consequently, stabilized Cdt1 continually primes DNA re-replication during the $\mathrm{S}$ phase and induces genomic instability characterized by aneuploidy (39). Consistent with this finding, data from the present study showed that the tumor-derived D1- $\Delta 266-295$ mutation triggered Cdt1 stabilization during the $\mathrm{S}$ phase in cell culture, and induced a greater accumulation of $>4 \mathrm{~N}$ cells than wild-type cyclin D1. Nuclear D1- $266-295$, but not wild-type cyclin D1, is capable of inhibiting Cdt1 proteolysis and promoting re-replication, which is consistent with a previously published study whose findings indicated that overexpressed wild-type cyclin D1 does not induce a DNA damage response $(40,41)$. The above observations suggest that the genomic instability triggered by nuclear retention of the active cyclin D1/CDK complex is a crucial determinant to elicit the oncogenicity of cyclin D1 in addition to its prevalent overexpression in cancers. However, the underlying molecular mechanism remains to be determined.

In conclusion, the results provided by the present study suggest that the features of constitutive nuclear localization of this tumor-derived cyclin D1- $\Delta 266-295$ mutant, contribute to the genesis and progression of neoplastic growth. Results of the present study are likely to expand knowledge of the oncogenicity of constitutively active cyclin D1 mutant proteins. However, further investigation into the role played by the nuclear cyclin D1/CDK complex in the context of genomic instability and neoplastic transformation is required.

\section{Acknowledgements}

This study was supported by grants from the National Natural Science Foundation of China (no. 30771099), and the National S and T Major Project for Infectious Diseases (2009ZX10004-903).

\section{References}

1. Kato J, Matsushime H, Hiebert SW, Ewen ME and Sherr CJ: Direct binding of cyclin $\mathrm{D}$ to the retinoblastoma gene product $(\mathrm{pRb})$ and $\mathrm{pR} b$ phosphorylation by the cyclin $\mathrm{D}$-dependent kinase CDK4. Genes Dev 7: 331-342, 1993.

2. Lundberg AS and Weinberg RA: Functional inactivation of the retinoblastoma protein requires sequential modification by at least two distinct cyclin-cdk complexes. Mol Cell Biol 18: 753-761, 1998.

3. Weinberg RA: The retinoblastoma protein and cell cycle control. Cell 81: 323-330, 1995.

4. Coqueret O: Linking cyclins to transcriptional control. Gene 299: 35-55, 2002.

5. Bienvenu F, Jirawatnotai S, Elias JE, et al: Transcriptional role of cyclin D1 in development revealed by a genetic-proteomic screen. Nature 463: 374-378, 2010.

6. Fu M, Rao M, Bouras T, et al: Cyclin D1 inhibits peroxisome proliferator-activated receptor gamma-mediated adipogenesis through histone deacetylase recruitment. J Biol Chem 280: 16934-16941, 2005.

7. Gladden $\mathrm{AB}$ and Diehl JA: Location, location, location: the role of cyclin D1 nuclear localization in cancer. J Cell Biochem 96: 906-913, 2005.

8. Deshpande A, Sicinski P and Hinds PW: Cyclins and cdks in development and cancer: a perspective. Oncogene 24: 2909-2915, 2005.

9. Gillett C, Smith P, Gregory W, et al: Cyclin D1 and prognosis in human breast cancer. Int J Cancer 69: 92-99, 1996.

10. Sicinski P, Donaher JL, Parker SB, et al: Cyclin D1 provides a link between development and oncogenesis in the retina and breast. Cell 82: 621-630, 1995.

11. Bartkova J, Lukas J, Strauss M and Bartek J: The PRAD-1/ cyclin D1 oncogene product accumulates aberrantly in a subset of colorectal carcinomas. Int J Cancer 58: 568-573, 1994.

12. Bartkova J, Lukas J, Muller H, Strauss M, Gusterson B and Bartek J: Abnormal patterns of D-type cyclin expression and G1 regulation in human head and neck cancer. Cancer Res 55: 949-956, 1995. 
13. Hibberts NA, Simpson DJ, Bicknell JE, et al: Analysis of cyclin D1 (CCND1) allelic imbalance and overexpression in sporadic human pituitary tumors. Clin Cancer Res 5: 2133-2139, 1999.

14. Hemmer S, Wasenius VM, Haglund C, et al: Deletion of 11q23 and cyclin D1 overexpression are frequent aberrations in parathyroid adenomas. Am J Pathol 158: 1355-1362, 2001.

15. Ikeguchi M, Sakatani T, Ueta T and Kaibara N: Cyclin D1 expression and retinoblastoma gene protein (pRB) expression in esophageal squamous cell carcinoma. J Cancer Res Clin Oncol 127: 531-536, 2001.

16. Jin $\mathrm{M}$, Inoue $\mathrm{S}$, Umemura $\mathrm{T}$, et al: Cyclin $\mathrm{D} 1$, p16 and retinoblastoma gene product expression as a predictor for prognosis in non-small cell lung cancer at stages I and II. Lung Cancer 34: 207-218, 2001

17. Alt JR, Cleveland JL, Hannink M and Diehl JA: Phosphorylationdependent regulation of cyclin D1 nuclear export and cyclin D1-dependent cellular transformation. Genes Dev 14: 3102-3114, 2000 .

18. Bodrug SE, Warner BJ, Bath ML, Lindeman GJ, Harris AW and Adams JM: Cyclin D1 transgene impedes lymphocyte maturation and collaborates in lymphomagenesis with the myc gene EMBO J 13: 2124-2130, 1994.

19. Lovec H, Grzeschiczek A, Kowalski MB and Moroy T: Cyclin $\mathrm{D} 1 / \mathrm{bcl}-1$ cooperates with myc genes in the generation of B-cel lymphoma in transgenic mice. EMBO J 13: 3487-3495, 1994.

20. Gladden AB, Woolery R, Aggarwal P, Wasik MA and Diehl JA: Expression of constitutively nuclear cyclin D1 in murine lymphocytes induces B-cell lymphoma. Oncogene 25: 998-1007, 2006.

21. Diehl JA, Zindy F and Sherr CJ: Inhibition of cyclin D1 phosphorylation on threonine-286 prevents its rapid degradation via the ubiquitin-proteasome pathway. Genes Dev 11: 957-972, 1997.

22. Germain D, Russell A, Thompson A and Hendley J: Ubiquitination of free cyclin D1 is independent of phosphorylation on threonine 286. J Biol Chem 275: 12074-12079, 2000.

23. Diehl JA, Cheng M, Roussel MF and Sherr CJ: Glycogen synthase kinase-3beta regulates cyclin D1 proteolysis and subcellular localization. Genes Dev 12: 3499-3511, 1998.

24. Lu F, Gladden AB and Diehl JA: An alternatively spliced cyclin D1 isoform, cyclin D1b, is a nuclear oncogene. Cancer Res 63 : 7056-7061, 2003.

25. Benzeno S, Lu F, Guo M, et al: Identification of mutations that disrupt phosphorylation-dependent nuclear export of cyclin D1. Oncogene 25: 6291-6303, 2006.

26. Moreno-Bueno G, Rodriguez-Perales S, Sanchez-Estevez C, et al: Cyclin D1 gene (CCND1) mutations in endometrial cancer. Oncogene 22: 6115-6118, 2003.

27. Cobrinik D: Pocket proteins and cell cycle control. Oncogene 24: 2796-2809, 2005.
28. Shinozaki H, Ozawa S, Ando N, et al: Cyclin D1 amplification as a new predictive classification for squamous cell carcinoma of the esophagus, adding gene information. Clin Cancer Res 2: $1155-1161,1996$

29. Shamma A, Doki Y, Shiozaki H, et al: Cyclin D1 overexpression in esophageal dysplasia: A possible biomarker for carcinogenesis of esophageal squamous cell carcinoma. Int J Oncol 16: 261-266, 2000.

30. Leveque C, Marsaud V, Renoir JM and Sola B: Alternative cyclin D1 forms $\mathrm{a}$ and $\mathrm{b}$ have different biological functions in the cell cycle of B lymphocytes. Exp Cell Res 313: 2719-2729, 2007.

31. Guo Y, Yang K, Harwalkar J, et al: Phosphorylation of cyclin D1 at Thr 286 during $\mathrm{S}$ phase leads to its proteasomal degradation and allows efficient DNA synthesis. Oncogene 24: 2599-2612, 2005.

32. Yang K, Guo Y, Stacey WC, et al: Glycogen synthase kinase 3 has a limited role in cell cycle regulation of cyclin D1 levels. BMC Cell Biol 7: 33, 2006

33. Alao JP, Stavropoulou AV, Lam EW, Coombes RC and Vigushin DM: Histone deacetylase inhibitor, trichostatin A induces ubiquitin-dependent cyclin D1 degradation in MCF-7 breast cancer cells. Mol Cancer 5: 8, 2006.

34. Feng Q, Sekula D, Muller R, Freemantle SJ and Dmitrovsky E: Uncovering residues that regulate cyclin $\mathrm{D} 1$ proteasomal degradation. Oncogene 26: 5098-5106, 2007.

35. Agami R and Bernards R: Distinct initiation and maintenance mechanisms cooperate to induce $\mathrm{G} 1$ cell cycle arrest in response to DNA damage. Cell 102: 55-66, 2000.

36. Alao JP: The regulation of cyclin D1 degradation: roles in cancer development and the potential for therapeutic invention. Mol Cancer 6: 24, 2007.

37. Lin DI, Lessie MD, Gladden AB, Bassing $\mathrm{CH}$, Wagner $\mathrm{KU}$ and Diehl JA: Disruption of cyclin D1 nuclear export and proteolysis accelerates mammary carcinogenesis. Oncogene 27: 1231-1242, 2008.

38. Truong $\mathrm{LN}$ and $\mathrm{Wu} \mathrm{X}$ : Prevention of DNA re-replication in eukaryotic cells. J Mol Cell Biol 3: 13-22, 2011.

39. Aggarwal P, Lessie MD, Lin DI, et al: Nuclear accumulation of cyclin D1 during S phase inhibits Cul4-dependent Cdt1 proteolysis and triggers p53-dependent DNA rereplication. Genes Dev 21: 2908-2922, 2007.

40. Spruck CH, Won KA and Reed SI: Deregulated cyclin E induces chromosome instability. Nature 401: 297-300, 1999.

41. Tort F, Bartkova J, Sehested M, Orntoft T, Lukas J and Bartek J: Retinoblastoma pathway defects show differential ability to activate the constitutive DNA damage response in human tumorigenesis. Cancer Res 66: 10258-10263, 2006. 